\title{
Long-term survival after intraperitoneal chemotherapy with paclitaxel-cisplatin for recurrent primary peritoneal cancer resistant to multiple lines of intravenous chemotherapy
}

\author{
Hyejeong Hue ${ }^{1}$, Kidong Kim², HyoJin Kim³ ${ }^{3}$ Dong Hoon Suh², Jae Hong No², Yong Beom Kim² \\ ${ }^{1}$ Department of Obstetrics and Gynecology, Seoul National University Hospital, Seoul; Departments of ${ }^{2}$ Obstetrics and Gynecology, ${ }^{3}$ Pathology, Seoul \\ National University Bundang Hospital, Seongnam, Korea
}

The long-term survival of heavily pretreated patients with primary peritoneal cancer (PPC) is uncommon. Here, we report on a patient with PPC refractory to multiple lines of intravenous chemotherapy, namely, a combined regimen of paclitaxel and carboplatin, and single regimens of topotecan, docetaxel, cisplatin, and gemcitabine. However, after intraperitoneal (IP) chemotherapy with paclitaxel-cisplatin, the patient's condition improved, and she has been progression-free for more than 4 years. Interestingly, before the IP chemotherapy, the recurrences were limited to the peritoneal cavity. These results suggest that IP recurrence might be a predictor of a good response to IP chemotherapy.

Keywords: Peritoneal neoplasms; Neoplasm recurrence, local; Infusions, parenteral; Drug therapy; Ovarian neoplasms

\section{Introduction}

Ovarian cancer was estimated to have the fourth highest incidence of all cancers among Korean women aged 15-34 years in 2017 [1,2]. According to the Korea Statistical Office, the incidence of ovarian cancer has been increasing annually [2]. Primary peritoneal cancer (PPC) is a rare cancer, being similar in several aspects to epithelial ovarian cancer (EOC). Similar to EOC patients, many patients with PPC are diagnosed at advanced stages, and recurrences are common after treatment. The standard treatment for advanced EOC and PPC is debulking surgery followed by taxane-platinum chemotherapy, administered intravenously. Approximately $10-15 \%$ of advanced EOC patients achieve and maintain response to treatment. However, in most patients, the disease remains persistent after therapy or relapses occur [3]. Finally, complete recovery is difficult in patients with recurrent EOC and PPC. Therefore, the aim of the treatment is to increase the patient's life expectancy and improve their quality of life via palliative care.

In intraperitoneal (IP) chemotherapy, drugs are administrated directly into the peritoneal cavity through a catheter.
The theoretical advantages of IP chemotherapy are: 1) drugs with high local concentrations may penetrate into small tumor nodules, and drug resistance in cells may be overcome; 2 ) prolonged exposure time may enhance the activity of certain classes of drugs; and 3) systemic toxicities may be reduced, allowing for greater tolerance to treatment [4]. In the initial treatment of EOC and PPC, IP chemotherapy is associated with a better survival than is intravenous (IV) chemotherapy. However, in cases of recurrence, the advantage of IP over IV chemotherapy is still unclear.

Received: 2018.02.09. Revised: 2019.02.07. Accepted: 2019.02.11. Corresponding author: Kidong Kim

Department of Obstetrics and Gynecology, Seoul National University Bundang Hospital, 82 Gumi-ro 173-beon-gil, Bundanggu, Seongnam 13620, Korea

E-mail: kidong.kim.md@gmail.com

https://orcid.org/0000-0001-9254-6024

Articles published in Obstet Gynecol Sci are open-access, distributed under the terms of the Creative Commons Attribution Non-Commercial License (http://creativecommons. org/licenses/by-nc/3.0/) which permits unrestricted non-commercial use, distribution, and reproduction in any medium, provided the original work is properly cited.

Copyright $\odot 2019$ Korean Society of Obstetrics and Gynecology 


\title{
Obstetrics \& Gynecology Science
}

\author{
Vol. 62, No. 4, 2019
}

In this study, we report a case of PPC refractory to 5 lines of IV chemotherapy. However, after IP chemotherapy with paclitaxel-cisplatin, the patient showed improvement, and has been progression-free for more than 4 years. This study suggests that IP chemotherapy can be effective for patients with recurrent EOC or PPC.

\section{Case report}

A 52-year-old woman visited a local gynecological hospital. An ultrasound and computed tomography (CT) revealed a pelvic cystic mass, $8.1 \times 5.3 \mathrm{~cm}$ in size, with peritoneal seeding (Fig. 1). The cancer antigen 125 serum level was $52 \mathrm{U} / \mathrm{mL}$ (normal range, 0-35 U/mL). She was transferred to the Seoul National University Bundang Hospital for further evaluation and treatment (Fig. 1).

The laparotomy revealed a pelvic tumor, $8 \mathrm{~cm}$ in diameter, with irregular margins. It was adherent to the peritoneum, and did not invade the right ovary. A large amount of ascites was also observed. Moreover, seeding nodules were found in the pararectal space, pelvic peritoneum, and sigmoid, ascending, and descending colon. The patient's gynecologic oncologist performed a hysterectomy, bilateral salpingooophorectomy, bilateral pelvic lymph node dissection, and supracolic omentectomy, removing all gross tumor lesions. After surgery, no gross residual tumor remained. The his-

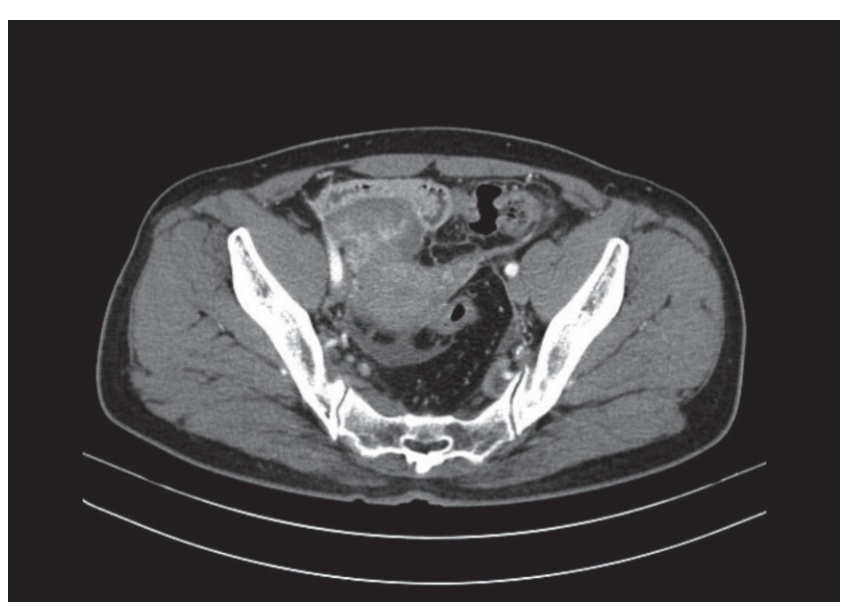

Fig. 1. Computed tomography images before the first debulking surgery, revealing a solid tumor on the right ovary, $8.1 \times 5.3 \mathrm{~cm}$ in size, with diffuse peritoneal thickening, and infiltration of a small amount of ascites in the pelvic cavity and within both paracolic gutters. tological examination revealed a poorly differentiated PPC; therefore, this tumor was categorized as a high-grade serous carcinoma. The tumor stage was IIIB.

After the first surgery, the patient received 6 cycles of IV paclitaxel and carboplatin. However, she experienced a peritoneal recurrence after a month, and underwent secondary debulking surgery for tumor removal from the abdominal wall, peritoneum, bladder, and bowel serosa. She then received 3 cycles of IV topotecan. The chemotherapy regimen was chosen based on the physician's preference because there are no known differences between drug efficacy in platinum-resistant ovarian cancer. However, immediately after topotecan chemotherapy, the patient experienced another peritoneal recurrence. She received 3 cycles of IV docetaxel, but the recurrent tumor progressed. As the patient was young and in good overall health, the treatment was continued to increase life expectancy. A tertiary debulking surgery was performed, and the histological examination confirmed PPC recurrence. She then received 3 cycles of IV cisplatin. However, after a month, the patient experienced another peritoneal recurrence in the distal small bowel, and seeding nodules were identified in the pelvic cavity. After subsequent administration of 3 cycles of IV gemcitabine, the development of peritoneal seeding nodules was observed (Fig. 2A). The fourth debulking surgery included a small bowel resection and anastomosis, and mass excision from the small bowel and bilateral pelvic wall. Additionally, an IP port for chemotherapy was inserted. Following this procedure, no gross residual tumor remained. Subsequently, the patient received 6 cycles of IP chemotherapy with paclitaxel and cisplatin, with the following protocol: paclitaxel $135 \mathrm{mg} / \mathrm{m}^{2}$ IV (D1), cisplatin $75 \mathrm{mg} / \mathrm{m}^{2} \mathrm{IP}(\mathrm{D} 2)$, and paclitaxel $80 \mathrm{mg} / \mathrm{m}^{2}$ IP (D8).

There has been no recurrence for 4 years and 6 months since the end of IP chemotherapy, and we believe that the patient has been cured (Fig. 2B).

\section{Discussion}

To our knowledge, this is the first patient with refractory PPC to achieve long-term recurrence-free survival over 4 years after IP chemotherapy. In addition, the response of the refractory PPC to the IP chemotherapy is unique, considering that the advantage of IP chemotherapy over IV chemotherapy is 


\section{Obstetrics \& Gynecology Science}

Hyejeong Hue, et al. Paclitaxel-cisplatin for recurrent PCC

unclear for cases of recurrence.

The prognosis of heavily pretreated patients with EOC or PPC is poor. The response rates to the following agents are low: topotecan, 20\%; gemcitabine, 19\%; liposomal doxorubicin, 26\%; oral etoposide, 27\%; docetaxel, $22 \%$; and weekly paclitaxel, 21\% [5]. Additionally, in the AURELIA trial, the addition of bevacizumab improved the progression-free survival (PFS) and overall survival (OS); however, the median PFS remained 6.7 months [6]. Therefore, the PFS of over 4 years in the present case is noteworthy.

Many trials have shown the advantage of IP chemotherapy as first-line treatment for EOC [7-9]. For example, in the Gynecologic Oncology Group (GOG) 172 trial, the OS of women with stage III cancer was higher by 16 months after IP chemotherapy than after IV chemotherapy (65.6 vs. 49.7 months, $P=0.03$ ) [6]. However, other studies showed insufficient evidence to support this [10]. Specifically, in the GOG 252 trial, no significant difference was found in the PFS between the patients receiving IP and IV chemotherapy [11]. Nevertheless, these results demonstrated an overall advantage of IP chemotherapy for EOC and PPC. Although IP treatment is associated with improved survival, it has not been widely adopted as a standard of care in both cancer types because of concerns about excessive toxicity, difficult logistics, and cost. Therefore, we had considered IP treatment as a final option.

Numerous phase I and II trials have evaluated the effect of IP chemotherapy for cases of recurrence using various drugs. Overall, the response rates to chemotherapy varied from $10 \%$ to $60 \%$; in pathologically confirmed cases, the rates were up to $35 \%$, and in patients with microscopic residual disease, between $40 \%$ and $60 \%$ [12]. Nonetheless, the effect of IP chemotherapy on survival remains uncertain because of the lack of data from randomized trials for cases of recurrence. Altogether, these results suggest that IP chemotherapy may be effective only in a subset of patients, rather than in all patients with EOC or PPC. Therefore, it is important to identify this subset of patients.

It is not clear if a biomarker can be used to identify this subset of patients. One study showed that a decreased BRCA1 expression was associated with an improved response to IP chemotherapy [13], in contrast to the tumor expression of LMAN2, FZD4, FZD5, or STT3A. Moreover, low expression of APC2 and high expression of FUT9 were associated with lower OS after IP than after IV chemotherapy $(P \leq 0.007)$ [14]. In this study, BRCA and other genes were not tested for biomarker potential because gene analysis at the time of the case occurrence was not covered by insurance, and thus was not universally performed.

Approximately $40 \%$ of first recurrences in patients treated with IP chemotherapy occur outside the peritoneal cavity [15]. Even if the first recurrence is IP, most patients eventually experience extra-peritoneal recurrence. However, in this case, all the 5 progressions were restricted to the peritoneal cavity.

The fact that this PPC, which does not respond to IV chemotherapy, showed a good response to IP chemotherapy suggests that the tumor was localized and the local concentration of chemotherapeutic drugs was higher, although
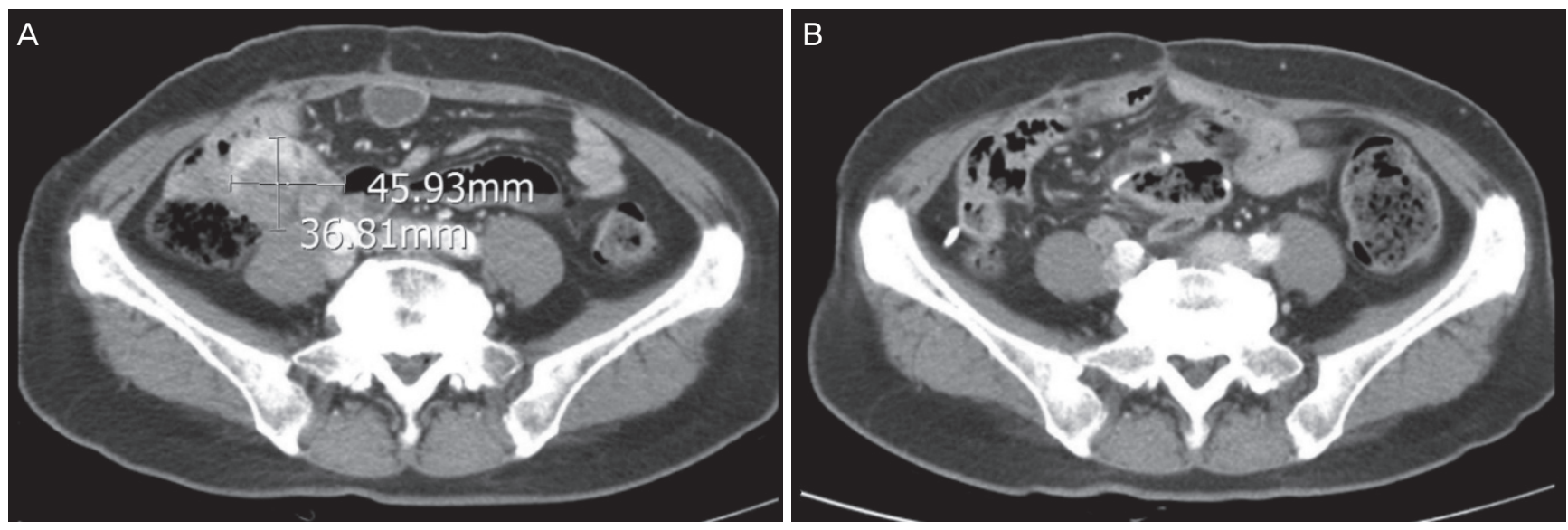

Fig. 2. Pelvic computed tomography images showing $(A)$ a size increase in 2 peritoneal seeding nodules after 3 cycles of intravenous gemcitabine, in November 2012; (B) absence of new lesions after 6 cycles of intraperitoneal chemotherapy, in March 2013. 


\section{Obstetrics \& Gynecology Science}

Vol. 62, No. 4, 2019

more evidence is needed to support this hypothesis. In this case report, recurrences were limited to the peritoneal cavity, and the recurrent lesions, which were 2 to $3 \mathrm{~mm}$ in diameter or smaller, may have experienced significantly higher drug exposure due to IP administration than they would with IV administration. These lesions can serve as a biomarker of a good response to IP chemotherapy.

\section{Conflict of interest}

No potential conflict of interest relevant to this article was reported.

\section{Ethical approval}

The study was approved by the Institutional Review Board of Seoul National University Bundang Hospital (IRB No. B-1804/462-701) and performed in accordance with the principles of the Declaration of Helsinki.

\section{Patient consent}

The patient consent was waived by the Institutional Review Board

\section{References}

1. Jung KW, Won YJ, Kong HJ, Lee ES. Prediction of cancer incidence and mortality in Korea, 2018. Cancer Res Treat 2018; 50:317-23.

2. Statistics Korea. Cancer registry statistics [Internet]. Daejeon: Statistics Korea; 2018 [cited 2018 Feb 8]. Available from: http://kosis.kr.

3. Armstrong DK. Relapsed ovarian cancer: challenges and management strategies for a chronic disease. Oncologist 2002;7 Suppl 5:20-8.

4. Vermorken JB. Intraperitoneal chemotherapy in advanced ovarian cancer: recognition at last. Ann Oncol 2006;17 Suppl 10:x241-6.

5. Morgan RJ Jr, Armstrong DK, Alvarez RD, BakkumGamez JN, Behbakht K, Chen LM, et al. Ovarian cancer, version 1.2016, NCCN Clinical Practice Guidelines in Oncology. J Natl Compr Canc Netw 2016;14:1134-63.

6. Pujade-Lauraine E, Hilpert F, Weber B, Reuss A, Poveda A, Kristensen $\mathrm{G}$, et al. Bevacizumab combined with chemotherapy for platinum-resistant recurrent ovarian cancer: the AURELIA open-label randomized phase III trial. J Clin Oncol 2014;32:1302-8.

7. Alberts DS, Liu PY, Hannigan EV, O'Toole R, Williams SD, Young JA, et al. Intraperitoneal cisplatin plus intravenous cyclophosphamide versus intravenous cisplatin plus intravenous cyclophosphamide for stage III ovarian cancer. N Engl J Med 1996;335:1950-5.

8. Armstrong DK, Bundy B, Wenzel L, Huang HQ, Baergen $\mathrm{R}$, Lele $\mathrm{S}$, et al. Intraperitoneal cisplatin and paclitaxel in ovarian cancer. N Engl J Med 2006;354:34-43.

9. Markman M, Bundy BN, Alberts DS, Fowler JM, ClarkPearson DL, Carson LF, et al. Phase III trial of standarddose intravenous cisplatin plus paclitaxel versus moderately high-dose carboplatin followed by intravenous paclitaxel and intraperitoneal cisplatin in small-volume stage III ovarian carcinoma: an intergroup study of the Gynecologic Oncology Group, Southwestern Oncology Group, and Eastern Cooperative Oncology Group. J Clin Oncol 2001;19:1001-7.

10. Yen MS, Juang CM, Lai CR, Chao GC, Ng HT, Yuan CC. Intraperitoneal cisplatin-based chemotherapy vs. intravenous cisplatin-based chemotherapy for stage III optimally cytoreduced epithelial ovarian cancer. Int I Gynaecol Obstet 2001;72:55-60.

11. Walker J, Brady MF, DiSilvestro PA, Fujiwara K, Alberts D, Zheng $W$, et al. A phase III trial of bevacizumab with IV versus IP chemotherapy for ovarian, fallopian tube, and peritoneal carcinoma: an NRG Oncology Study. Gynecol Oncol 2016;141 Suppl 1:208.

12. Vermorken JB. The role of intraperitoneal chemotherapy in epithelial ovarian cancer. Int J Gynecol Cancer 2000;10:26-32.

13. Lesnock JL, Darcy KM, Tian C, Deloia JA, Thrall MM, Zahn C, et al. BRCA1 expression and improved survival in ovarian cancer patients treated with intraperitoneal cisplatin and paclitaxel: a Gynecologic Oncology Group Study. Br J Cancer 2013;108:1231-7.

14. Seagle BL, Eng KH, Yeh JY, Dandapani M, Schiller E, Samuelson $\mathrm{R}$, et al. Discovery of candidate tumor biomarkers for treatment with intraperitoneal chemothera- 


\section{Obstetrics \& Gynecology Science}

Hyejeong Hue, et al. Paclitaxel-cisplatin for recurrent PCC

py for ovarian cancer. Sci Rep 2016;6:21591.

15. Esselen KM, Rodriguez N, Growdon W, Krasner C, Horowitz NS, Campos S. Patterns of recurrence in ad- vanced epithelial ovarian, fallopian tube and peritoneal cancers treated with intraperitoneal chemotherapy. Gynecol Oncol 2012;127:51-4. 